\title{
East Meets West in the Western Carpathians: Contact Zone Between Alpine and Carpathian Caddisfly Lineages Evidenced by COI Barcodes
}

Jana Bozáňová

Comenius University in Bratislava

Fedor Čiampor Jr

Slovak Academy of Sciences

Tomasz Mamos

University of Łódź

Michal Grabowski

University of Łódź

Zuzana Čiamporová-Zat'ovičová ( $\square$ zuzana.zatovicova@savba.sk)

Slovak Academy of Sciences

\section{Research Article}

Keywords: biodiversity, freshwater fauna, European biota, BIN_W, Western Carpathians

Posted Date: July 9th, 2021

DOl: https://doi.org/10.21203/rs.3.rs-678096/v1

License: (c) (1) This work is licensed under a Creative Commons Attribution 4.0 International License. Read Full License 


\section{Abstract}

European mountains are important areas regarding biodiversity of the continent, and they also harbour diverse freshwater fauna, which is critically endangered in terms of the current species extinction. However, sufficient knowledge of this valuable part of European biota is no longer possible without molecular data. This study focuses on the genetic diversity and distribution patterns of the classical representative of the mountain freshwater fauna, caddisfly Rhyacophila tristis, in the Western Carpathians. Based on the $\mathrm{COI}$ mitochondrial marker, two genetic lineages (separate BINs) were identified. BIN_W was found in 16 localities in the western part of the study area, BIN_E in 44 eastern localities. The data obtained indicate that BIN_W occurs in a significantly narrower altitude range, BIN_E is more closely related to the Balkan populations than to co-occurring BIN_W, and that the contact zone of the lineages passes through the Western Carpathians. The study revealed phylogeographic and demographic differences between lineages, supporting hypothesis of their evolutionary independence and specific ecological preferences.

The obtained genetic data shifted our knowledge on the populations of the studied caddisfly and suggested patterns that could be common to other freshwater species. This could help us to protect unique freshwater ecosystems and preserve European biodiversity.

\section{Introduction}

Population genetic studies are inevitable for understanding the mechanisms of distribution and dispersion of species under the influence of past climate changes. The results acquired may subsequently help to estimate evolution of species affected by current climate change, as they provide valuable information on the size of the gene pool or the degree of species adaptation in relation to environmental changes (Manel et al., 2003; Storfer et al., 2010; Alp et al., 2012; Mamos et al., 2014; Mamos et al., 2016; Grabowski et al., 2017; Copilaş-Ciocianu et al., 2018; Copilaş-Ciocianu, Zimţa \& Petrusek, 2019; Wattier et al., 2020).

Recent molecular data on aquatic and terrestrial taxa show that the Western Carpathians (W Carpathians) constitute an important European biodiversity hotspot (Neumann et al., 2005; Kotlík et al., 2006; Theissinger et al., 2012; Vörös et al., 2016; Copilaș-Ciocianu et al., 2017; Juřičková et al., 2017; Mamos et al., 2021). Some studies also document major role of the mountain ranges as a glacial refugia for various species or genetic lineages (Pinceel et al., 2005; Magri et al., 2006; Jamrichová, Potůčková \& Horsák, 2014; Wielstra, Babik \& Arntzen, 2015; Mamos et al., 2016; Mráz \& Ronikier, 2016; CopilașCiocianu et al., 2017; Jamrichová, Petr \& Jiménez-Alfaro, 2017). The W Carpathians form a huge reservoir of freshwater, including a rich system of submountain and mountain springs and streams, which are still understudied in the context of the genetic diversity and population structure of their inhabitants. Nevertheless, few studies on aquatic mountain species show that populations of a single species inhabiting separate regions of the W Carpathians can be significantly different from the rest of Europe in 
respect to their genetic diversity (Pauls, Lumbsch \& Haase, 2006; Pauls et al., 2009; Lehrian, Pauls \& Hasse, 2009).

The knowledge on the spatial distribution of genetic diversity at a species level is an essential prerequisite for understanding the resilience of biota in the changing environment across geological periods (Lande \& Shannon, 1996; Frankham, Briscoe \& Ballou, 2002). This is especially important for species inhabiting specific biotopes such as mountain aquatic ecosystems. The caddisfly Rhyacophila tristis Pictet, 1834 is one of the three species from the Rhyacophila tristis group, widespread in Europe. Adults of $R$. tristis are relatively good fliers, which guarantees efficient dispersal. They prefer upper parts of streams, because larvae require well-oxygenated water with low levels of organic matter (Robert \& Curtean-Bănăduc, 2005). Bálint et al. (2011) provided the first data on the large-scale population genetics of this (sub)montane species on the European level. The study revealed strong genetic differences between populations from the western and eastern part of Europe, but due to sparse sampling and large distances between sites, such a result was very likely. It was suggested that $R$. tristis survived in an independent Pleistocene refugia both in the Alps and the Carpathians. Unfortunately, the area of the W Carpathians was not covered in this study and so the data from an important part of the species area of distribution was missing.

Western Carpathians represent the northernmost part of the Alpine-Carpathian mountain chain (Bielik, 1999). Due to high habitat diversity of this area, including numerous montane springs and streams, we hypothesize that the genetic patterns and level of the genetic diversity found by Bálint et al. (2011) at the large geographic scale could be also detected at a much smaller area of the W Carpathians. It could also be expected that this mountain range may include a contact zone between the Alpine (western) and Carpathian (eastern) lineages of the $R$. tristis. To test these assumptions, we generated and analysed a large dataset of $R$. tristis samples from the springs and streams in the W Carpathians and adjacent areas, which also allowed us to fill the gap in the phylogeographic puzzle of this species in the European mountains.

\section{Results}

The caddisfly Rhyacophila tristis had a relatively wide distribution in the studied area of the Carpathians, both in streams and springs. The BIN algorithm and ASAP analysis resulted in identical partitioning of the 5 ' COI dataset into two MOTUs, whose distribution was largely disjunct (Fig. 2A).

The MOTU1, equal to BIN BOLD:AAD5574 (BIN_W), was recorded at 16 localities in the west, while the MOTU2, equal to BIN BOLD:ADL4166 (BIN_E), was found at 44 localities in the east. Both BINs were found only at two localities in the Fatra-Tatra area: the Biela Voda stream (T180) and Jazierce (V033). In the Fatra-Tatra area, where both BINs met, the highest levels of molecular divergence and a relatively clear boundary between the two BINS (MOTUs) were found (Fig. 2A).

The comparison of the altitude range in the distribution of both BINs revealed significant differences. The BIN_W occurred at a significantly narrower altitude interval compared to BIN_E ( $p$-value $<0.05$; Fig. 2B). 
On the other hand, no statistically significant preference for the habitat type (spring vs. stream) was detected for either BIN.

The haplotype network revealed the relationships between the two BINs identified in the study area as well as their connection with two additional $R$. tristis BINs from other European mountain ranges: the Balkan BIN_B (BOLD:ADL4367) occurring, e.g., in Rila Mts in Bulgaria and the BIN_A (BOLD:AAD5573) documented from the Swiss and Italian Alps (Fig. 2C). In total, $28 \mathrm{COI}$ haplotypes of $R$. tristis were identified within 161 individuals from the W Carpathians. The BIN_W included 59 sequences grouped in 11 haplotypes, BIN_E 102 sequences in 17 haplotypes. There were found no significant differences in molecular genetic indices between the two BINs (Table 1).

Both BIN_W and BIN_E showed similar haplotype network patterns, i.e., star-like topology with a central, the most-frequent haplotype. RT01 was the most common haplotype in the BIN_W lineage, and was shared with German, Austrian, and Italian localities. The BIN_W included eight private haplotypes, six of them were found only in the Fatra-Tatra Area and two were private for the Western Beskids. Within the BIN_E, the total number of private haplotypes was 14 , while four private haplotypes were identified in the Fatra-Tatra Area, the Slovak Ore Mts, and the Vihorlat Mts, one was found in the Poloniny Mts and the Apuseni Mts. The most common haplotype of the BIN_E (RT12 with 55 sequences) occurred at 32 sites. Only a single haplotype (RT06) was shared among the Inner and Outer Eastern Carpathians (PM, VM, LB) and more distanced localities in Romania (Fig. 2C). Sequences from Romania were assigned to BIN_E, while sequences from Germany and Austria fell into the western BIN_W. The Bayesian phylogenetic reconstruction of $R$. tristis $\left(5^{\prime} \mathrm{COI}\right)$ revealed that the divergence of the species likely started some 2.5 million years ago (Myr) and the western and eastern BINs diverged ca one million years later. Possible misidentification of sequences (marked as R. pubescens) downloaded from databases was also noted within the analysis (Fig. 2D). Both phylogenetic trees based on $3^{\prime}$ or $5^{\prime} \mathrm{COI}$ sequences also showed that the BIN_E is more closely related to the Balkan BIN_B from the remote Rila Mts than to the BIN_W occurring in the same mountain system (Figs. 2D, S1).

The AMOVA of the whole dataset showed that most of the molecular variance could be attributed to the division of samples to geomorphological units or to river basins (Table S2). After partitioning the dataset into BIN_W and BIN_E, the highest proportion of the molecular variance was found within sampling localities (= subpopulations) in both BINs (Table 2). In the BIN_W, a relatively large portion of the total variance was explained by the differences among subpopulations within GU / RB. In the BIN_W, the variation at this level was negligible, however, influence of the division into GU / RB was suggested (Table 2).

The $\mathrm{F}_{\mathrm{ST}}$ values indicate a similar range of genetic differentiation within both BINs (Fig. 3). They showed that the most localities are relatively well connected in both BINs, but some level of isolation could not be refused among some subpopulations. The tests of isolation by distance revealed a positive correlation (Mantel test: $r=0.601 ; p$-value $=0.001)$, supported also by the significant spatial autocorrelation ( $p$-value 
$=0.000 ;$ Fig. S2). Such a positive and statistically significant correlation suggests a structuring effect of the geographical distance among studied localities.

The mismatch distribution analysis suggests a recent population expansion for both BIN_W and BIN_E, a scenario depicted by a typical unimodal distribution (Fig. 4A). In the BIN_W, this was also supported by the non-significant value of the Harpending's raggedness index $(r)$. The non-significant values of the Harpending's raggedness index ( $r$ ) was also found for BIN_E subpopulations of springs and streams separately (Fig. 5A). However, the mismatch distribution graph for BIN_E of streams has the character of a population expansion, as evidenced by neutrality tests that have statistically significant negative values (Table 3). In the case of BIN_E springs, all values of the neutrality tests were negative, which indicates a population expansion, but only the result of the Fu's Fs test was significant (Table 3). The values of Tajima's D, Fu's Fs and Fu and Li's D neutrality tests for both BINs were negative and statistically significant (Table 3), which corresponds well with the Mismatch distribution test and supports population expansion hypothesis in both BINs (Fig. 4A). Overall, the results of the neutrality tests of the entire $R$. tristis population have negative values and the values of the Fu's Fs and Fu and Li's D tests are statistically significant (Table 3).

The eBSP also indicates population expansion in both $R$. tristis BINs in the study area (Fig. 4B), which likely started before ca 50-80 ka in BIN_W and ca 25-30 ka in BIN_E. While the population of BIN_W grew gradually, the BIN_E population size increased rapidly in the last 20-25 ka (Fig. 4B). In the case of BIN_E, the expansion was found in populations of both springs and streams (Fig. 5B). The resulting eBSP plots show sudden population expansion and twice the present effective population size of BIN_E in streams in contrast to springs, where the trend of population expansion was gradual and not so prominent (Fig. 5B).

\section{Discussion}

Rhyacophila tristis is an upstream species (Céréghino, Cugny \& Lavandier, 2002) requiring welloxygenated water with low organic matter levels (Robert \& Curtean-Bănăduc, 2005). The results of the first European phylogeographic study on this species by Bálint et al. (2011) showed strong genetic differences between its western and eastern populations, with lineages that probably survived independently in the Pleistocene refugia in the Alps and in the Carpathians. The presence of the western (BIN_W) and the eastern lineage (BIN_E) was also detected in a much smaller area of the W Carpathians. Moreover, based on the genetic landscape approach, we proved that W Carpathians form a contact zone between these BINs. Reconstruction of phylogeny suggests that the two BINs separated relatively long ago ( 2 Myr, late Pleistocene), indicating that in fact they may represent two separate species. The analysis also showed that the eastern BIN_E is more closely related to the Balkan samples (BIN_B, Bulgarian Rila Mts) than to the BIN_W co-occuring in the same mountain system (W Carpathians). The climatic conditions in the mid and late Pleistocene impacted the geographical distribution of the species (Janeau \& Aulagnier, 1997), which could also lead to intraspecific differentiation or even speciation. When most of the mountains were covered by ice, some populations of species moved from mountains 
to adjacent lowland areas and some of them stayed in the isolated areas with suitable environmental conditions. The study of Asellus aquaticus (Isopoda) also confirms the survival of populations in the periglacial areas (Sworobowicz et al., 2020). However, to make a sound conclusion, additional studies including more molecular markers would be needed.

Concerning altitude, BIN_W occurred at a significantly narrower altitude interval compared to BIN_E, where the altitude of the localities ranged from approximately 200 to $1700 \mathrm{~m}$ a.s.l. Generally, elevation gradients shape the dispersal and population structure in mountain species due to isolation of high-elevation sites, local adaptation to extreme environments, or both (Hughes et al., 2008). Distribution of freshwater species can be also very often explained by altitude, but also flow type or different ecological demands (Illies \& Botosaneanu, 1963). Possible relationship between the altitude range and the distribution scale of $R$. tristis BINs in our study area was indicated since the BIN_E was more widespread (44 localities) unlike the BIN_W, which was constrained to a smaller area (16 localities). However, it cannot be ruled out that BIN_W is extended further to the west, because two 3' COI sequences of Balint et al. (2009) from the Western Alps demonstrably belong to BIN_W (Fig. S1). If other samples analysed in the future confirm this assumption, it is likely that BIN_W could also have a wider altitudinal range.

Many freshwater species possess endemic genetic lineages or at least private haplotypes occuring in different European mountain systems, including the W Carpathians. For example, the caddisfly Drusus discolor very likely persisted in the Tatra Mts in numerous refugia over multiple glacial cycles (Pauls, Lumbsch \& Haase, 2006). Biodiversity richness of the W Carpathians is also supported by studies of the cold-adapted gammarids Gammarus balcanicus (Mamos et al., 2014; Mamos et al., 2016), G. fossarum (Copilaș-Ciocianu et al., 2017) or G. jazdzewskii (Rudolph et al., 2018). Although no endemic lineages of $R$. tristis were found in the W Carpathians, we have detected the presence of 21 private haplotypes within BIN_W and BIN_E, which have not yet been confirmed anywhere else.

The AMOVA of the whole W Carpathian population of $R$. tristis revealed distinct variation among geomorphological units or river basins. This is however very likely due separate phylogeography, i.e., history and distribution of both detected BINs. The results of AMOVA on the level of individual BINs were more consistent with respect to GU or RB division, which suggests that considering BINs as separate evolutionary units is more natural. In both BINs, the highest portion of the variability was assigned to differences within subpopulations (localities), suggesting relatively recent colonization of the studied area. In the western BIN_W, however, reasonable part of the genetic variability was attributed to differences among subpopulations within geomorphological units / river basins, similarly as in Elmis aenea from springs of the same area (Bozáňová et al. 2020). On the other hand, AMOVA of BIN_E revealed no variability among localities within GU/RB, but slight differences between GU/RB were detected. This was similar to the more fluvial riffle beetle Limnius perrisi from the same study or the blackfly Simulium degrangei (Jedlička et al., 2012) studied also in the W Carpathians. $F_{S T}$ values of both BINs agreed with the results of AMOVA. The differences between W Carpathian $R$. tristis lineages in the amount of genetic variation between localities within the GU/RB could indicate that the degree of local isolation is lower and / or the dispersal ability could be higher in BIN_E than in BIN_W. The eastern lineage 
also occurred in a significantly wider range of altitudes compared to western one. Altitude influences a series of other factors, such as the proportion of suitable habitats or migration patterns, and indirectly it shapes species population genetics (Manel et al., 2003). Moreover, high-elevation sites are often reservoirs of specific part of the species' genetic diversity (Hughes, 2007; Finn et al., 2011; Vuataz et al., 2016), but their vulnerability is high and the loss of isolated populations from higher elevations could result in a loss of important, locally adapted genotypes (Schiffers et al., 2013). Vice versa, adaptation potential and long-term survival of species or genetic lineages are dependent on sufficient intraspecific genetic diversity (Spielman, Brook \& Frankham, 2004; Frankham, 2005), and from this perspective BIN_E could be at an advantage because it looks to be more tolerant to a wider scale of altitudes, and thus also environmental conditions. Differences between lineages (BINs) and, conversely, similar patterns of distribution of genetic variability with, e.g., riffle beetles from the same area but also with other insect species (Bunn \& Hughes, 1997; Hughes et al., 1998) confirm the need to study population genetics in detail at the level of natural evolutionary units. It may allow us to better understand the evolution and phylogeography of individual taxa, or to look for general trends in the development of fauna of entire ecosystems.

Beside genetic variability distribution, the demography of the $R$. tristis BINs differs too in the W Carpathians. The BIN_W started to expand roughly around 40-50 thousand years ago (ka), while BIN_E around 25 ka during the Last Glacial Maximum (LGM). At that time, latitudinal temperature gradient existed across Europe, when winter soil temperatures were $10-20^{\circ} \mathrm{C}$ colder in Central and Northern Europe and around $2-4^{\circ} \mathrm{C}$ in Southern Europe than today (Barron \& Pollard, 2002). Such conditions could suit the up-stream caddisfly $R$. tristis, and so its glacial expansion could be expected (Bennet \& Provan, 2008). The population of the W Carpathian BIN_E grew sharply compared to BIN_W, and its present effective population size is around five times higher. We assume that this difference could be linked to the different distribution of the BINs in terms of altitude. BIN_E with a significantly wider altitude range probably found higher potential for dispersal in various mountain ranges within the W Carpathians. In addition, when comparing the eBSP of spring and stream habitats of BIN_E, a gradual, longer lasting increase in springs was observed. This could be explained by stable environmental conditions (and thus also longer time available for survival) in springs regardless of climatic changes (Minshall \& Winger, 1968; Odum, 1971; Butler \& Hobbs, 1982; Cushing \& Wolf, 1984; Glazier \& Gooch, 1987; Gooch \& Glazier, 1991). Differences in eBSP and AMOVA could indicate that BIN_W survived in the study area for a longer period and that genetic drift occurred after the invasion of individual sites, causing genetic differences among populations within the GU/RB. In contrast, for BIN_E, the results suggest that it spread to the W Carpathians more abruptly and relatively rapidly after LGM. Subtle differences in the variability among GU/RB suggest that individual units/basins were colonised by a part of the originally homogeneous population, which brought specific, although not significantly different compositions of haplotypes. However, the shorter time of occurrence in the studied area has not yet allowed BIN_E to create differences between local populations, as very likely happened within BIN_W.

\section{Conclusion}


The analysis of the caddisfly $R$. tristis of springs and streams in the Western Carpathians showed that these habitats form an important and unique part of the freshwater ecosystems producing or harbouring reasonable intraspecific genetic diversity, and it can be assumed that these habitats play an equal role in other areas. The comparison with co-occurring species revealed very interesting results regarding the differences or similarities of their genetic diversity as well as history. This study did not have the ambition to address the taxonomy of the studied species, but it is clear that more attention should be paid to this, because, as genetic and geographical data have suggested, the possibility that the two identified lineages (BINs) represent separate cryptic species cannot be ruled out. The eastern lineage (BIN_E) most likely colonized the W Carpathians from Southeast Europe, probably at the end of the LGM, while BIN_W appears to originate in areas west of the W Carpathians but it survived and developed its population in the study area for a longer time. What is certain, however, is that the W Carpathians is the place where both lineages meet. What role do W Carpathian springs and streams play in survival and preservation of genetic diversity of $R$. tristis, whether the lineages hybridize in the contact zone or what are their specific ecological requirements, will need to be further studied including additional samples from a wider area of distribution or other genetic markers.

Nevertheless, the data gained shifted our knowledge on the populations of the studied caddisfly and suggested patterns that could be common to other freshwater species. Such information could be feasible for protection of aquatic ecosystems and preserving European freshwater biodiversity.

\section{Materials And Methods}

\section{Study area}

The study area includes the W Carpathians, one of the major geomorphological units of the Carpathian mountain range (Fig. 1A). It occupies approximately one-third of the total area of the Carpathians (Kondracki, 1989). Most of the W Carpathian ranges are of moderate altitude, ranging mostly from 500 to $1300 \mathrm{~m}$ a.s.l., only sparsely exceeding $1500 \mathrm{~m}$ a.s.I. (Kondracki, 1989). Geologically, the W Carpathians are diversified, especially in the inner part of the arc, with various kinds of bedrock (Mesozoic limestones and dolomites, Paleozoic granites and metamorphic rocks or Tertiary volcanic rocks). The outer W Carpathians are built almost solely from flysch (Grecula, 1997). During the Pleistocene cycles, the area of the W Carpathians remained mostly unglaciated. The continental ice sheet was only located in the northernmost foothills of the Polish part during its maximal extent in the Middle Pleistocene and the mountain glaciers covered valleys in the highest ranges (above $1700 \mathrm{~m}$ a.s.I.) (Lukniš, 1964). The mountain glaciers completely disappeared around 8.500 years ago (Lindner et al., 2003). The precipitation in the area is determined by differences in altitude and geomorphological relief. Hydrologically, the W Carpathian rivers have a rain-snow regime with floods in springs and summers.

The studied localities include 15 springs and 43 streams situated mainly in Slovakia, partially in Czechia and Poland, within the Inner and Outer Western Carpathian geomorphological units/subunits. These 
localities were supplemented by sampling sites in the Inner Eastern Carpathians (Vihorlat Mts - VM, Poloniny Mts - PM) and the Outer Eastern Carpathians (Low Beskids - LB) (Fig. 1B; Table S1).

\section{Sampling and morphological identification}

The qualitative sampling of macrozoobenthos was performed in 2016-2017, within the framework of a broader hydrobiological research focused on karst springs and diversity of the W Carpathian streams. It was carried out by a multi-habitat kick-sampling technique (Frost, 1971), using a hydrobiological handnet with a mesh size of $0.5 \mathrm{~mm}$. In the field, specimens were fixed in $96 \%$ ethanol. In the laboratory, the invertebrates were sorted into higher taxonomic groups using stereomicroscope, prefixed with clean ethanol and stored in a freezer at $-25^{\circ} \mathrm{C}$. Samples were supplemented by additional specimens of $\mathrm{R}$. tristis from the collection of the Plant Science and Biodiversity Centre, Slovak Academy of Sciences (Bratislava). The individuals of R. tristis were morphologically identified using the determination keys Sedlák (1980) and Waringer \& Graf (2011).

\section{DNA extraction and PCR amplification}

DNA was extracted from a total of 161 individuals, two legs of each individual, using the Chelex protocol (Casquet, Thebaud \& Gillespie, 2012), followed by PCR amplification of ca. 650 bp-long barcoding fragment of the mitochondrial cytochrome c oxidase subunit I (5' COI) using the primer pair LCO1490 HCO2198 (Folmer et al., 1994). The PCR was performed in a total volume of $25 \mu$ containing $5 \mu$ of $5 \times$ DreamTaq $^{\text {TM }}$ Buffer, $1.5 \mu \mathrm{l}$ of $\mathrm{Mg}^{+2}(25 \mathrm{mM}), 0.5 \mu$ l of each primer (concentration $\left.5 \mathrm{mM}\right), 0.5 \mu \mathrm{l}$ of dNTP Mix $(20 \mathrm{mM}), 0.125 \mu \mathrm{l}(0.625 \mathrm{U})$ DreamTaq DNA Polymerase, $11.875 \mu \mathrm{l}$ ultra-pure $\mathrm{H}_{2} \mathrm{O}$ and $5 \mu \mathrm{l}$ of DNA template. The PCR cycling consisted of a 2-min initial denaturation at $94^{\circ} \mathrm{C}$, followed by 40 cycles of $94^{\circ} \mathrm{C}$ (40 s) denaturation, $46^{\circ} \mathrm{C}(40 \mathrm{~s})$ annealing and $72^{\circ} \mathrm{C}(1 \mathrm{~min})$ extension and termination at $72^{\circ} \mathrm{C}(10 \mathrm{~min})$ for a final extension. A $4 \mu \mathrm{l}$ aliquot of the PCR products were visualized by GoldView (Solarbio) in electrophoresis on a $1 \%$ agarose gel and GelLogic imaging equipment to check PCR product quality and length. The PCR products were purified with Exo-FastAP Thermo Scientific and were sent for sequencing to Macrogen Europe Inc., Amsterdam.

Additionally, the non-barcoding 3' fragment of the COI was amplified using primers Jerry and Pat (Simon et al., 1994) from six selected individuals (see below for rationale) to create a phylogenetic tree together with 19 sequences (456 bp-long) of $R$. tristis and 15 sequences of congeneric species $R$. aquitanica McLachlan, 1879 (8) and R. carpathica Botoșaneanu, 1995 (7) from Bálint et al. (2009; 2011) (Fig. S1).

\section{Data analysis}

The obtained sequences were edited in SEQUENCHER v5.1 and aligned using the MUSCLE algorithm (Edgar, 2004) in MEGA v7 (Kumar, Stecher \& Tamura, 2016). In total, the 5' COI dataset consisted of 194 sequences of $R$. tristis. It included 161 sequences from the Western Carpathians (46 localities), the Poloniny Mts (3 loc.), the Vihorlat Mts (7 loc.) and Low Beskids (1 loc.) (Fig. 1). They were supplemented by 33 reference sequences of $R$. tristis from outside the W Carpathians ( 5 - Austria, 6 - Bulgaria, 5 Germany, 2 - Italy, 11 - Romania, 4 - Switzerland), used for the reconstruction of haplotype networks. 
These sequences were obtained from the BOLD (http://www.boldsystems.org). All sequences used in this study are included in the publicly available BOLD dataset DS-SKRHYTRI.

Two methods were used to test $R$. tristis samples for presence of the deeper lineages or speciesequivalents. The first was BIN algorithm in BOLD (https://v4.boldsystems.org), where every uploaded sequence is compared to all records and assigned to an existing or a newly created Barcode Index Number (BIN) (Ratnasingham \& Hebert, 2007). The BIN system clusters are unique and include molecular operational taxonomic units (MOTU) that closely correspond to the species. The second approach utilized the Assemble Species by Automatic Partitioning (ASAP), a novel and powerful method to build species partitions, based on pairwise genetic distances from single locus sequence alignments (i.e., barcode data sets) (Puillandre, Brouillet \& Achaz, 2021).

The haplotype data files and the diversity indices were generated in DnaSP v5.10 (Librado \& Rozas, 2009) based on $5^{\prime} \mathrm{COI}$ data. We calculated haplotype diversity $(H)$, nucleotide diversity $(\pi)$, number of polymorphic sites (S) and average number of nucleotide differences $(\mathrm{K})$ per individual BINs of $R$. tristis species within the W Carpathians. Statistical comparison of the genetic indices between BINs was computed with the Wilcoxon signed rank test for paired data in R v4.0.2 (http://www.r-project.org). The same test was used to compare the altitude range between the individual BINs of the study area.

Haplotype networks were reconstructed using the median-joining method (MJN) in PopART v1.7 (Leigh \& Bryant, 2015). The networks include sequences outside the W Carpathians to explain the phylogeographic relationships and haplotype distribution in the broader context.

The phylogeny was reconstructed based on 5' COI haplotypes using Bayesian approach in BEAST v2.5 (Bouckaert et al., 2019) and, separately, for the 3' COI marker also including published sequences (Bálint et al., 2009; Bálint et al., 2011). As an outgroup, the European congeners $R$. aquitanica and $R$. carpathica were included. The nucleotide substitution model was set through bModelTest (Bouckaerd \& Drummond, 2017). The tree prior was set to Birth-Death and a strict molecular clock was used following the Path Sampling Selection. The strict molecular clock was calibrated with the standard mitochondrial rate for arthropod COI equal to 0.0115 substitutions/site/Myr (Brower, 1994). Two runs of Markov chain Monte Carlo (MCMC), each 20 million iterations long and sampled every 1,000 iterations, were performed for both fragments. Runs were examined using Tracer v1.7 (Rambaut et al., 2018), and all sampled parameters achieved a sufficient sample size (ESS $>200$ ). Tree files were combined using Log-Combiner v2.5.2 (Bouckaert et al., 2019), with the removal of the non-stationary $25 \%$ burn-in phase. The maximum clade credibility chronogram was generated using TreeAnnotator v2.5.2 (Bouckaert et al., 2019).

The spatial diversity pattern in the studied area of Carpathians was illustrated with a genetic landscape approach generated with Alleles in Space (AIS; Miller, 2005). The genetic landscape visualizes the abrupt transitions between populations and groups of populations characterized by divergent haplotypes. First, using the AIS software, genetic distances between localities were calculated based on all 5 ' $\mathrm{COI}$ sequences and connected into a network based on the Delaunay Triangulation. The genetic distance values were set in the midpoints of each connection in the network. The raw genetic distances acquired 
were interpolated afterwards and the matrix of the 'elevation' values, with their respective latitude and longitude coordinates, was then imported into QGIS 3.18 (https://qgis.osgeo.org) software to produce a genetic divergence surface image using the inverse distance weighted algorithm. The resulting image was plotted onto a relief map to create a final map in which the hypsometric tints (red-blue) reflect the genetic distance between population pairs.

To test if spatial distance is structuring the molecular diversity of $5^{\prime} \mathrm{CO}$ fragments, we run two types of isolation by distance tests: Mantel test (Mantel, 1967) and general spatial autocorrelation test using Alleles in Space (AIS; Miller, 2005). Both tests analyse correlation between spatial and molecular distance. To assess the significance, tests were run with 1,000 permutations.

The population structure of $R$. tristis in the W Carpathians based on $5^{\prime}$ COI was characterized by the analysis of molecular variance (AMOVA) and fixation indices $\left(F_{S T}\right)$ using Arlequin v3.5 (Excoffier \& Lischer, 2010). The AMOVA was used to estimate whether the observed genetic diversity may be attributed to the geographical or river basin partitioning of populations in three levels: among geomorphological units (GU) / river basins (RB), among sampling sites within GU / RB and within sampling sites. We also performed AMOVA and $\mathrm{F}_{\mathrm{ST}}$ separately for individual BINs of the $R$. tristis, to find out the differences between them. 138 sequences of $R$. tristis (36 localities) were included to calculate the $F_{S T}$ index, localities with 1 sequence were excluded. To test the significance of covariance components and fixation indices, 1,000 permutations were performed.

Historical expansion patterns of the R. tristis in the studied area of Carpathians, based on $5^{\prime} \mathrm{COI}$ were examined in three different approaches. First, Tajima's D (Tajima, 1989), Fu's Fs (Fu, 1997) and Fu and Li's D (Fu \& Li, 1993) neutrality tests with 10,000 permutations were calculated in DnaSP to test the selective neutrality and population stability. Secondly, nucleotide mismatch distribution was performed in Arlequin v3.5 (Excoffier \& Lischer, 2010) to detect the demographic and spatial dynamics of population expansion history in the $\mathrm{W}$ Carpathians. The fit between the observed and expected distributions was evaluated by the test statistics of goodness-of-fit, including the sum of squared deviation (SSD) and Harpending's raggedness index ( $r$ ). Additionally, because part of the individuals was found in multiple springs and streams, the mentioned approaches were also performed separately for both habitats.

Finally, the extended Bayesian skyline plot (eBSP), implemented in BEAST v2.6.2 software package (Bouckaert et al., 2019), was used to show the fluctuations of $R$. tristis demography in the W Carpathians over time. The model of substitution and molecular clock were set up identical as in the case of the phylogeny reconstruction. For comparison, two runs of Monte Carlo Markov Chains (MCMC) were performed, each 40 million iterations long and sampled every 10,000 iterations. The runs were examined in Tracer v1.7 and all the parameters reached the effective sampling size (ESS) above 200. The eBSP plots were produced using R v4.0.2 software (http://www.r-project.org). Both runs showed complementary results, thus only one is presented.

\section{Declarations}


Acknowledgements

We would like to thank the research team of the Department of Ecology, Comenius University in Bratislava, who performed fieldwork with us. This study was partially supported by the Slovak National Grant Agency VEGA 2/0084/21 and VEGA 1/0127/20.

\section{Author information}

\section{Affiliations}

ZooLab, Plant Science and Biodiversity Centre, Slovak Academy of Sciences, Bratislava, Slovak Republic Jana Bozáňová, Fedor Čiampor Jr, Zuzana Čiamporová-Zat'ovičová

Department of Ecology, Faculty of Natural Sciences, Comenius University in Bratislava, Bratislava, Slovak Republic

Jana Bozáňová, Zuzana Čiamporová-Zatovičová

Department of Invertebrate Zoology and Hydrobiology, Faculty of Biology \& Environmental Protection, University of Łódź, Łódź, Poland

Tomasz Mamos, Michal Grabowski

\section{Contributions}

J.B, F.Č.Jr, Z.ČZ., T.M., M.G. designed the study. J.B, F.Č.Jr, Z.ČZ. collected material. J.B. performed the laboratory works. J.B, F.Č.Jr, Z.ČZ., T.M., M.G. designed data analysis. J.B, T.M. performed analyses on the data. J.B. prepared figures and/or tables. All authors reviewed the manuscript.

Corresponding author

Correspondence to Zuzana Čiamporová-Zat'ovičová

Additional information

Competing interests

The authors declare no competing interests.

\section{Data availability}

All sequences used in this study are included in the publicly available BOLD dataset DS-SKRHYTRI.

After receiving the manuscript, we will complete the DOI. 


\section{References}

1. Alp, M., Keller, I., Westram, A. M., Robinson, C.T. How river structure and biological traits influence gene flow: a population genetic study of two stream invertebrates with differing dispersal abilities. Freshw. Biol. 57, 969-981. https://doi.org/10.1111/j.1365-2427.2012.02758.x (2012).

2. Barron, E. \& Pollard, D. High-resolution climate simulations of oxygen isotope stage 3 in Europe. Quat. Res. 28, 296-309. https://doi.org/10.1006/qres.2002.2374 (2002).

3. Bálint, M., Botoşaneanu, L., Ujvárosi, L., Popescu, O. Taxonomic revision of Rhyacophila aquitanica (Trichoptera: Rhyacophilidae), based on molecular and morphological evidence and change of taxon status of Rhyacophila aquitanica ssp. carpathica to Rhyacophila carpathica stat. $\mathrm{n}$. Zootaxa 2148, 39-48. https://doi.org/10.11646/zootaxa.2148.1.3 (2009).

4. Bálint, M., Ujvárosi, L., Dénes, A. L., Octavian, P. European phylogeography of Rhyacophila tristis Pictet (Trichoptera: Rhyacophilidae): preliminary results. Zoosymposia 5, 11-18. https://doi.org/ 10.11646/zoosymposia.5.1.1 (2011).

5. Bennet, K. \& Provan, J. What do we mean by "refugia"? Quat. Sci. Rev. 27, 2449-2455, DOI: (2008).

6. Bielik, M. Geophysical features of the Slovak Western Carpathians. Geol. Q. 43, 251-262. https://doi.org/10.1016/j.quascirev.2008.08.019 (1999).

7. Bouckaert, R. R. \& Drummond, A. J. bModelTest: Bayesian phylogenetic site model averaging and model comparison. BMC Evol. Biol. 17(42). https://doi.org/10.1186/s12862-017-0890-6 (2017).

8. Bouckaert, R., Vaughan, T. G., Barido-Sottani, J., Duchêne, S., Fourment, M., Gavryushkina, A., Heled, J., Jones, G., Kühnert, D., De Maio, N., Matschiner, M., Mendes, F.K. , Müller, N. F., Ogilvie, H. A., duPlessis, L., Popinga, A., Rambaut, A., Rasmussen, D., Siveroni, I., Suchard, M. A., Wu, C-H., Xie, D., Zhang, C., Stadler, T., Drummond, A. J. 2019. BEAST 2.5: an advanced software platform for Bayesian evolutionary analysis. PLoS Comput. Biol. 15(4), e1006650. https://doi.org/ 10.1371/journal.pcbi.1006650 (2019).

9. Bozáňová, J., Čiamporová-Zat'ovičová, Z., Čiampor Jr, F., Mamos, T., Grabowski, M. The tale of springs and streams: how different aquatic ecosystems impacted the mtDNA population structure of two riffle beetles in the Western Carpathians. PeerJ 8, e10039. https://doi.org/10.7717/peerj.10039 (2020).

10. Brower, A. V. Z. Rapid morphological radiation and convergence among races of the butterfly Heliconius erato inferred from patterns of mitochondrial DNA evolution. PNAS 91(14), 6491-6495. https://doi.org/10.1073/pnas.91.14.6491 (1994).

11. Bunn, S. E.\& Hughes, J. M. Dispersal and recruitment in streams: evidence from genetic studies. J N AM BENTHOL SOC 16, 338-346. https://doi.org/10.2307/1468022 (1997).

12. Butler, M. J. \& Hobbs, H. H. Drift upstream movement of invertebrates in a springbrook community ecosystem. Hydrobiologia 89, 153-159. https://doi.org/10.1007/BF00006168 (1982).

13. Casquet, J., Thebaud, C. \& Gillespie, R. G. Chelex without boiling, a rapid and easy technique to obtain stable amplifiable DNA from small amounts of ethanol-stored spiders. Mol 12(1), 136- 
141. https://doi.org/10.1111/j.1755-0998.2011.03073.x (2012).

14. Céréghino, R., Cugny, P. \& Lavandier, P. Influence of intermittent hydropeaking on the longitudinal zonation patterns of benthic invertebrates in a mountain stream. Int. Rev. Hydrobiol. 87, 47-60. https://doi.org/10.1002/1522-2632(200201)87:1<47::AID-IROH47>3.0.C0;2-9 (2002).

15. Copilaș-Ciocianu, D., Rutová, T., Pařil, P., Petrusek, A. 2017. Epigean gammarids survived millions of years of severe climatic fluctuations in high latitude refugia throughout the Western Carpathians. Mol. Phylogenet. Evol. 112, 218-229. https://doi.org/10.1016/j.ympev.2017.04.027 (2017).

16. Copilaş-Ciocianu, D., Zimţa, A. A., Grabowski, M., Petrusek, A. Survival in northern microrefugia in an endemic Carpathian gammarid (Crustacea: Amphipoda). Zool. Scr. 47, 357-372. https://doi.org/10.1111/zsc.12285 (2018).

17. Copilaș-Ciocianu, D., Zimța, A. \& Petrusek, A. Integrative taxonomy reveals a new Gammarus species (Crustacea, Amphipoda) surviving in a previously unknown southeast European glacial refugium. J ZOOL SYST EVOL RES 57, 272-297. https://doi.org/10.1111/jzs.12248 (2019).

18. Cushing, C. E \& Wolf, E. G. Primary production in Rattlesnake Springs, a cold desert spring-stream. Hydrobiologia 114, 229-236 (1984).

19. Edgar, R. C. MUSCLE: multiple sequence alignment with high accuracy and high throughput. Nucleic Acids Res. 32, 1792-1797. https://doi.org/10.1093/nar/gkh340 (2004).

20. Excoffier, L. \& Lischer, H. E. Arlequin suite ver 3.5: a new series of programs to perform population genetics analyses under Linux and Windows. Mol 10, 564-567. https://doi.org/10.1111/j.17550998.2010.02847.x (2010).

21. Finn, D. S., Bonada, N., Múrria, C., Hughes, J. M. Small but mighty: headwaters are vital to stream network biodiversity at two levels of organization. J N AM BENTHOL SOC 30, 963-980. https://doi.org/10.1899/11-012.1 (2011).

22. Folmer, O., Black, M., Hoeh, W., Lutz, R., Vrijenhoek, R. DNA primers for amplification of mitochondrial cytochrome c oxidase subunit I from diverse metazoan invertebrates. Mol Mar Biol Biotechnol 3(5), 294-299 (1994).

23. Frankham, R., Briscoe, D. A. \& Ballou, J. D. Introduction to Conservation Genetics. Cambridge: Cambridge University Press, UK 617 pp. (2002).

24. Frankham, R. Genetics and extinction. Biol. Conserv. 126,131-140, DOI: (2005).

25. Frost, S. 1971. Evaluation of kicking technique for sampling stream bottom fauna. Can. J. Zool. 49, 161-173. https://doi.org/10.1016/j.biocon.2005.05.002 (1971).

26. Fu, Y. X. Statistical tests of neutrality of mutations against population growth, hitchhiking and background selection. Genetics 147(2), 915-925 (1997).

27. Fu, Y. X. \& Li, W. H. Statistical tests of neutrality of mutations. Genetics 14, 693-709, DOI: (1993).

28. Glazier, D. S. \& Gooch, J. L. Macroinvertebrate assemblages in Pennsylvania (U.S.A.) springs. Hydrobiologia 150, 33-43 (1987). 
29. Gooch, J. L. \& Glazier, D. S. Temporal and spatial patterns in mid-Appalachian springs. Mem Ent Soc Can 155, 29-49 (1991).

30. Grabowski, M., Mamos, T., Bacela-Spychalska, K., Rewicz, T., Wattier, R. A. Neogene paleogeography provides context for understanding the origin and spatial distribution of cryptic diversity in a widespread balkan freshwater amphipod. PeerJ 5, e3016. https://doi.org/ 10.7717/peerj.3016 (2007).

31. Grecula, P. (ed.). Geological evolution of the Western Carpathians. Monograph: Mineralia Slovaca (1997).

32. Hughes, J. M., Bunn, S. E., Hurwood, D. A., Cleary, C. Dispersal and recruitment of Tasiagma ciliata (Trichoptera: Tasmiidae) in rainforest streams, south-east Queensland, Australia. Freshw. Biol. 41, 1-10. (1998).

33. Hughes, J. M. Constraints on recovery: using molecular methods to study connectivity of aquatic biota in rivers and streams. Freshw. Biol. 52, 616-631. https://doi.org/10.1111/j.13652427.2006.01722.x (2007).

34. Hughes, J. M., Schmidt, D. J., McLean, A., Wheatley, A. Population genetic structure in stream insects: what have we learned? p. 268-288. In: Aquatic Insects: challenges to populations: proceedings of the Royal Entomological Society's 24th symposium. (Eds. J. Lancaster \& R.A. Briers), CABI Publishing, Walllingford, U.K (2008).

35. Illies, J. \& Botosaneanu, L. Problèmes et méthodes de la classification et de la zonation écologique des eaux courantes, considérées surtout du point de vue faunistique. Mitteilungen der Internationalen Vereinigung für Limnologie 12, 1-57 (1963).

36. Jamrichová. E., Potůčková, A. \& Horsák, M. Landscape history, calcareous fen development historical events in the Slovak Eastern Carpathians. Veg. Hist. Archaeobot. 23, 497-513. https://doi.org/10.1007/s00334-013-0416-0 (2014).

37. Jamrichová, E., Petr, L. \& Jiménez-Alfaro, B. Pollen-inferred millennial changes in landscape patterns at a major biogeographical interface within Europe. J. Biogeogr. 44, 2386-2397, DOI: (2017).

38. Janeau, G. \& Aulagnier, S. Snow vole Chionomys nivalis (Martins 1842). Journal of Mountain Ecology 4, 1-11 (1997).

39. Jedlička, L., Kúdela, M., Szemes, T., Celec, P. Population genetic structure of Simulium degrangei (Diptera: Simuliidae) from Western Carpathians. Biologia 67, 777-787. https://doi.org/10.2478 /s11756-012-0057-2 (2012).

40. Juřičková, L., Pokorný, P., Hošek, J., Horáčková, J., Květoň, J., Zahajská, P., Jansová, A., Ložek, V. Early postglacial recolonisation, refugial dynamics the origin of a major biodiversity hotspot. A case study from the Malá Fatra mountains, Western Carpathians, Slovakia. The Holocene 28(4), 583-594. https://doi.org/10.1177/0959683617735592 (2017).

41. Kondracki, J. Karpaty. Wydanie drugie i poprawione [The Carpathians. Ed. 2]. - Wydawnictwa Szkolne i Pedagogiczne, Warszawa (1989). 
42. Kotlík, P., Deffontaine, V., Mascheretti, S., Zima, J., Michaux, J. R., Searle, J. B. A northern glacial refugium for bank voles (Clethrionomys glareolus). PNAS 103, 14860-14864. https://doi.org/ 10.1073/pnas.0603237103 (2006).

43. Kumar, S., Stecher, G. \& Tamura, K. MEGA7: Molecular Evolutionary Genetics Analysis Version 7.0 for Bigger Datasets. Mol. Biol 33, 1870-1874. https://doi.org/10.1093/molbev/msw054 (2016).

44. Lande, R. \& Shannon, S. 1996. The role of genetic variation in adaptation and population persistence in a changing environment. Evolution 216, 434-437 (1996).

45. Lehrian, S., Pauls, S. U. \& Haase, P. Contrasting patterns of population structure in the montane caddisflies Hydropsyche tenuis and Drusus discolor in the Central European highlands. Freshw. Biol. 54, 283-295. https://doi.org/10.1111/j.1365-2427.2008.02107.x (2009).

46. Leigh, J. W. \& Bryant, D. POPART: full-feature software for haplotype network construction. Methods Ecol. Evol. 6, 1110-1116. https://doi.org/10.1111/2041-210X.12410 (2015).

47. Librado, P. \& Rozas, J. DnaSP v5: a software for comprehensive analysis of DNA polymorphism data. Bioinformatics 25(11), 1451-1452. https://doi.org/10.1093/bioinformatics/btp187 (2009).

48. Lindner, L., Dzierzek, J., Marciniak, B., Nitychoruk, J. Outline of Quaternary glaciations in the Tatra Mts.: their development, age and limits. Geol. Q. 47, 269-280 (2003).

49. Lukniš, M. The course of the last glaciation of the Western Carpathians in the relation to the Alps, to the glaciation of northern Europe, and to the division of the central European Wurm into periods. Geografický Časopis 16, 127-142 (1964).

50. Magri, D., Fineschi, S., Bellarosa, R., Buonamici, A., Sebastiani, F., Schirone, B., Simeone, M. C., Vendramin, G. G. A new scenario for the Quaternary history of European beech populations: palaeobotanical evidence genetic consequences. New Phytol. 171, 199-221. https://doi.org/10.1111/ j.1469-8137.2006.01740.x (2006).

51. Mamos, T., Wattier, R., Majda, A., Sket, B., Grabowski, M. Morphological vs. molecular delineation of taxa across montane regions in Europe: the case study of Gammarus balcanicus Schäferna, 1922 (Crustacea: Amphipoda). J. Zool. Syst. Evol. Res. 52, 237-248. https://doi.org/10.1111/jzs.12062 (2014).

52. Mamos, T., Wattier, R., Burzýnski, A., Grabowski, M. The legacy of a vanished sea: a high level of diversification within a European freshwater amphipod species complex driven by 15 My of Paratethys regression. Mol. Ecol. 25, 795-810. https://doi.org/10.1111/mec.13499 (2016).

53. Mamos, T., Jazdzewski, K., Čiamporová-Zatovičová, Z., Čiampor, F., Grabowski, M. Fuzzy Species Borders of Glacial Survivalists Revealed Using a Multimarker Approach - A Case Study of Amphipod Crustaceans in the Carpathian Biodiversity Hotspot. Preprint from Research Square. https://doi.org/10.21203/ rs.3.rs-628030/v1 (2021).

54. Manel, S., Schwartz, M. K., Luikart, G., Taberlet, P. Landscape Genetics: combining landscape ecology and population genetics. Trends Ecol. Evol. 18, 189-197. https://doi.org/10.1016/S01695347(03)00008-9 (2003). 
55. Mantel, N. The detection of disease clustering and a generalized regression approach. Cancer Res. 27, 209-220 (1967).

56. Miller, M. P. Alleles In Space (AIS): Computer software for the joint analysis of interindividual spatial and genetic information. J. Hered. 96, 722-724. https://doi.org/10.1093/jhered/esi119 (2005).

57. Minshall, G. W. \& Winger, P. V. 1968. The Effect of Reduction in Stream Flow on Invertebrate Drift. Ecology 49, 580-582 (1968).

58. Mráz, P. \& Ronikier, M. Biogeography of the Carpathians: evolutionary spatial facets of biodiversity. Biol. J. Linn. Soc. 119, 528-559. https://doi.org/10.1111/bij.12918 (2016).

59. Neumann, K., Michaux, J. R., Maak, S., Jansman, H. A., Kayser, A., Mundt, G., Gattermann, R. Genetic spatial structure of European common hamsters (Cricetus cricetus) - a result of repeated range expansion and demographic bottlenecks. Mol. Ecol. 14, 1473-1483. https://doi.org/10.1111/ j.1365294X.2005.02519.x (2005).

60. Odum, E. P. Fundamentals of Ecology, Third Edition. Philadelphia: W.B. Saunders company (1971).

61. Pauls, S. U., Lumbsch, H. A. T. \& Haase, P. Phylogeography of the montane caddisfly Drusus discolor. evidence for multiple refugia and periglacial survival. Mol. Ecol.15(8), 2153-2169. https://doi.org/10.1111/j.1365-294X.2006.02916.x (2006).

62. Pauls, S. U., Theissinger, K., Ujvarosi, L., Bálint, M., Haase, P. Patterns of population structure in two closely related, partially sympatric caddisflies in eastern Europe: historic introgression, limited dispersal, and cryptic diversity. J N AM BENTHOL SOC 28, 517-536. https://doi.org/10.1899/08100.1 (2009).

63. Pinceel, J., Jordaens, K., Pfenninger, M., Backeljau, T. Rangewide phylogeography of a terrestrial slug in Europe: evidence for Alpine refugia rapid colonization after the Pleistocene glaciations. Mol. Ecol.14, 1133-1150. https://doi.org/10.1111/j.1365-294X.2005.02479.x (2005).

64. Puillandre, N., Brouillet, S. \& Achaz, G. ASAP: assemble species by automatic partitioning. Mol 21(2), 609-620. https://doi.org/10.1111/1755-0998.13281 (2021).

65. Rambaut, A., Drummond, A. J., Xie, D., Baele, G., Suchard, M. A. 2018. Posterior summarisation in Bayesian phylogenetics using Tracer 1.7. Syst. Biol. 67(5), 901-904. https://doi.org/10.1093/ sysbio/syy032 (2018).

66. Ratnasingham, S. \& Hebert, P. D. N. The barcode of life data system. Mol 7, 355-364. https://doi.org/ 10.1111/j.1471-8286.2007.01678.x (2007).

67. Robert, S. \& Curtean-Bănăduc, A. Aspects concerning Târnava Mare and Târnava Mică rivers (Transylvania, Romania) caddisfly (Insecta, Trichoptera) larvae communities. Transylv. Rev. Syst. Ecol. Res. 2, 89-98 (2005).

68. Rudolph, K., Coleman, C. O., Mamos, T., Grabowski, M. Description and post-glacial demography of Gammarus jazdzewskii sp. nov. (Crustacea: Amphipoda) from Central Europe. System. Biodivers. 16, 587-603. https://doi.org/10.1080/14772000.2018.1470118 (2018).

69. Sedlák, E. Řád Chrostíci - Trichoptera. In Rozkošný, R. (ed.), Klíc vodních larev hmyzu. ČSAV, Praha, 163-220 (1980). 
70. Schiffers, K., Bourne, E. C., Lavergne, S., Thuiller, W., Travis, J. M. Limited evolutionary rescue of locally adapted populations facing climate change. PHILOS T R SOC B 368, 20120083. https://doi.org/ 10.1098/rstb.2012.0083 (2013).

71. Simon, C., Frati, F., Beckenbach, A., Crespi, B., Liu, H., Flook, P. Evolution, weighting and phylogenetic utility of mitochondrial gene sequences and a compilation of conserved polymerase chain reaction primers. Ann. Entomol. Soc. Am. 87, 651-701 (1994).

72. Spielman, D., Brook, B. \& Frankham, R. Most species are not driven to extinction before genetic factors impact them. PNAS 101, 15261-15264. https://doi.org/10.1073/pnas.0403809101 (2004).

73. Storfer, A., Murphy, M. A., Spear, S. F., Holderegger, R., Waits, L. P. Landscape genetics: where are we now? Mol. Ecol.19, 3496-3514. https://doi.org/10.1111/j.1365-294X.2010.04691.x (2010).

74. Sworobowicz, L., Mamos, T., Grabowski, M., Wysocka, A. Lasting through the ice age: The role of the proglacial refugia in the maintenance of genetic diversity, population growth, and high dispersal rate in a widespread freshwater crustacean. Freshw. Biol. 65, 1028-1046. https://doi.org/ 10.1111/fwb.13487 (2020).

75. Tajima, F. The effect of change in population size on DNA polymorphism. Genetics 123(3), 597-601 (1989).

76. Theissinger, K., Bálint, M., Feldheim, K. A., Haase, P., Johannesen, J., Laube, I., Pauls, S. U. Glacial survival and post-glacial recolonization of an arctic-alpine freshwater insect (Arcynopteryx dichroa, Plecoptera, Perlodidae) in Europe. J. Biogeogr. 40, 236-248. https://doi.org/10.1111/j.13652699.2012.02793.x (2012).

77. Vörös, J., Mikulíček, P., Major, Á., Recuero, E., Arntzen, J. W. Phylogeographic analysis reveals northern refugia for the riverine amphibian Triturus dobrogicus (Caudata: Salamandridae). Biol. J. Linn. Soc. 119, 974-991. https://doi.org/10.1111/bij.12866 (2016).

78. Vuataz, L., Rutschmann, S., Monaghan, M. T., Sartori, M. Molecular phylogeny and timing of diversification in Alpine Rhithrogena (Ephemeroptera: Heptageniidae). BMC Evol. Biol. 16, 194. https://doi.org/10.1186/s12862-016-0758-1 (2016).

79. Waringer, J. \& Graf, W. Atlas of Central European Trichoptera Larvae: Atlas der Mitteleuropäischen Köcherfliegenlarven. Erik Mauch, Dinkelscherben, Germany, 468 pp. (2011).

80. Wattier, R., Mamos, T., Copilaş-Ciocianu, D., Jelić, M., Ollivier, A., Chaumot, A., Danger, M., Felten, V., Piscart, C., Žganec, K., Rewicz, T., Wysocka, A., Rigaud, T., Grabowski, M. Continental-scale patterns of hyper-cryptic diversity within the freshwater model taxon Gammarus fossarum (Crustacea, Amphipoda). Sci. Rep. 10, 16536. https://doi.org/10.1111/j.1365-2699.2012.02793.x (2020).

81. Wielstra, B., Babik, W., Arntzen, J. W. The crested newt Triturus cristatus recolonized temperate Eurasia from an extra-Mediterranean glacial refugium. Biol. J. Linn. Soc. 114, 574-587. https://doi.org/10.1111/bij.12446 (2015).

\section{Tables}


Table 1. Molecular diversity of BIN_W and BIN_E lineages of $R$. tristis population in the W Carpathians. The statistical significance was computed with the Wilcoxon signed rank test for paired data ( $p$-value).

\begin{tabular}{|lllll|}
\hline R. tristis & H & $\Pi$ & S & K \\
\hline BIN_W & 0.569 & 0.00136 & 9 & 0.781 \\
\hline BIN_E & 0.640 & 0.00142 & 16 & 0.816 \\
\hline BIN_E springs & 0.663 & 0.00140 & 5 & 0.808 \\
\hline BIN_E streams & 0.631 & 0.00141 & 12 & 0.812 \\
\hline Wilcoxon signed rank test & 103 & 117.5 & 114.5 & 117.5 \\
BIN_W/BIN_E (p-value) & $(0.33)$ & $(0.66)$ & $(0.58)$ & $(0.66)$ \\
\hline
\end{tabular}

$\mathrm{H}$ - haplotype diversity, $\Pi$ - nucleotide diversity, $\mathrm{S}$ - number of polymorphic sites, $\mathrm{K}$ - average number of nucleotide differences.

Table 2. Analysis of molecular variance (AMOVA) calculated from $1615^{\prime}$ COI mtDNA sequences of $R$. tristis (BIN_W, BIN_E) from springs and streams in the W Carpathians. The subpopulations are defined as individuals of one sampling site (see Table S1). 


\begin{tabular}{|c|c|c|c|c|c|c|c|}
\hline & Source of variation & Df & SS & $\begin{array}{l}\text { Variance } \\
\text { components }\end{array}$ & $\begin{array}{l}\% \text { of } \\
\text { variation }\end{array}$ & $F$ value & $\begin{array}{l}\mathrm{p}- \\
\text { value }\end{array}$ \\
\hline \multirow[t]{3}{*}{ GU } & Among GU & 2 & 2.383 & 0.01838 & 4.45 & $\begin{array}{l}\mathrm{F}_{\mathrm{CT}}= \\
0.044\end{array}$ & $\overrightarrow{0} .31$ \\
\hline & $\begin{array}{l}\text { Among subpopulations } \\
\text { within GU }\end{array}$ & 13 & 10.636 & 0.17115 & 41.40 & $\begin{array}{l}\mathrm{F}_{\mathrm{SC}}= \\
0.433\end{array}$ & $\overrightarrow{0.00}$ \\
\hline & Within subpopulations & 43 & 9.625 & 0.22384 & 54.15 & $\begin{array}{l}\mathrm{F}_{\mathrm{ST}}= \\
0.459\end{array}$ & $<$. \\
\hline \multirow[t]{3}{*}{ RB } & Among RB & 3 & 1.472 & -0.05425 & -14.16 & $\begin{array}{l}F_{C T}= \\
-0.142\end{array}$ & $\overrightarrow{0} .89$ \\
\hline & $\begin{array}{l}\text { Among subpopulations } \\
\text { within RB }\end{array}$ & 12 & 11.548 & 0.21350 & 55.73 & $\begin{array}{l}\mathrm{F}_{\mathrm{SC}}= \\
0.488\end{array}$ & $\overrightarrow{0.00}$ \\
\hline & Within subpopulations & 43 & 9.625 & 0.22384 & 58.43 & $\begin{array}{l}\mathrm{F}_{\mathrm{ST}}= \\
0.416\end{array}$ & $\dot{0.00}$ \\
\hline \multicolumn{8}{|c|}{ R. tristis BIN_E } \\
\hline & Source of variation & Df & SS & $\begin{array}{l}\text { Variance } \\
\text { components }\end{array}$ & $\begin{array}{l}\% \text { of } \\
\text { variation }\end{array}$ & F value & $\begin{array}{l}\mathrm{p}- \\
\text { value }\end{array}$ \\
\hline \multirow[t]{3}{*}{ GU } & Among GU & 7 & 7.667 & 0.06698 & 15.86 & $\begin{array}{l}\mathrm{F}_{\mathrm{CT}}= \\
0.159\end{array}$ & $\overrightarrow{0} 00$ \\
\hline & $\begin{array}{l}\text { Among subpopulations } \\
\text { within GU }\end{array}$ & 36 & 12.358 & -0.00986 & -2.33 & $\begin{array}{l}\mathrm{F}_{\mathrm{SC}}= \\
-0.02\end{array}$ & $\overrightarrow{0.65}$ \\
\hline & Within subpopulations & 58 & 21.181 & 0.36519 & 86.47 & $\begin{array}{l}\mathrm{F}_{\mathrm{ST}}= \\
0.135\end{array}$ & $<.09$ \\
\hline \multirow[t]{3}{*}{ RB } & Among RB & 6 & 7.505 & 0.06957 & 16.33 & $\begin{array}{l}\mathrm{F}_{\mathrm{CT}}= \\
0.163\end{array}$ & $\overrightarrow{0} .00$ \\
\hline & $\begin{array}{l}\text { Among subpopulations } \\
\text { within RB }\end{array}$ & 36 & 12.443 & -0.00878 & -2.06 & $\begin{array}{l}F_{S C}= \\
-0.025\end{array}$ & $>0.57$ \\
\hline & Within subpopulations & 58 & 21.181 & 0.36519 & 85.73 & $\begin{array}{l}\mathrm{F}_{\mathrm{ST}}= \\
0.143\end{array}$ & $<.07$ \\
\hline
\end{tabular}

Df - degree of freedom, SS - sum of squares, GU - geomorphological units, RB - river basins.

Table 3. $R$. tristis mtDNA COI 5 ' neutrality tests with p-values for the whole dataset, two different BINs, and springs and streams of the BIN_E. 


\begin{tabular}{|llll|}
\hline Species & Tajima's D test (p-value) & Fu's Fs test (p-value) & Fu and Li's D test (p-value) \\
\hline R. tristis & $0.29(0.71)$ & $-24.93(0.000)$ & $-4.033(<0.02)$ \\
\hline BIN_W & $-1.62(0.03)$ & $-4.788(<0.02)$ & $-5.153(<0.02)$ \\
\hline BIN_E & $-2.05(0.00)$ & $-2.804(<0.05)$ & $-2.751(<0.05)$ \\
\hline BIN_E springs & $-1.01(0.18)$ & $-2.355(0.00)$ & $-1.463(>0.10)$ \\
\hline BIN_E streams & $-1.87(0.01)$ & $-8.777(0.00)$ & $-1.866(<0.02)$ \\
\hline
\end{tabular}

Figures 


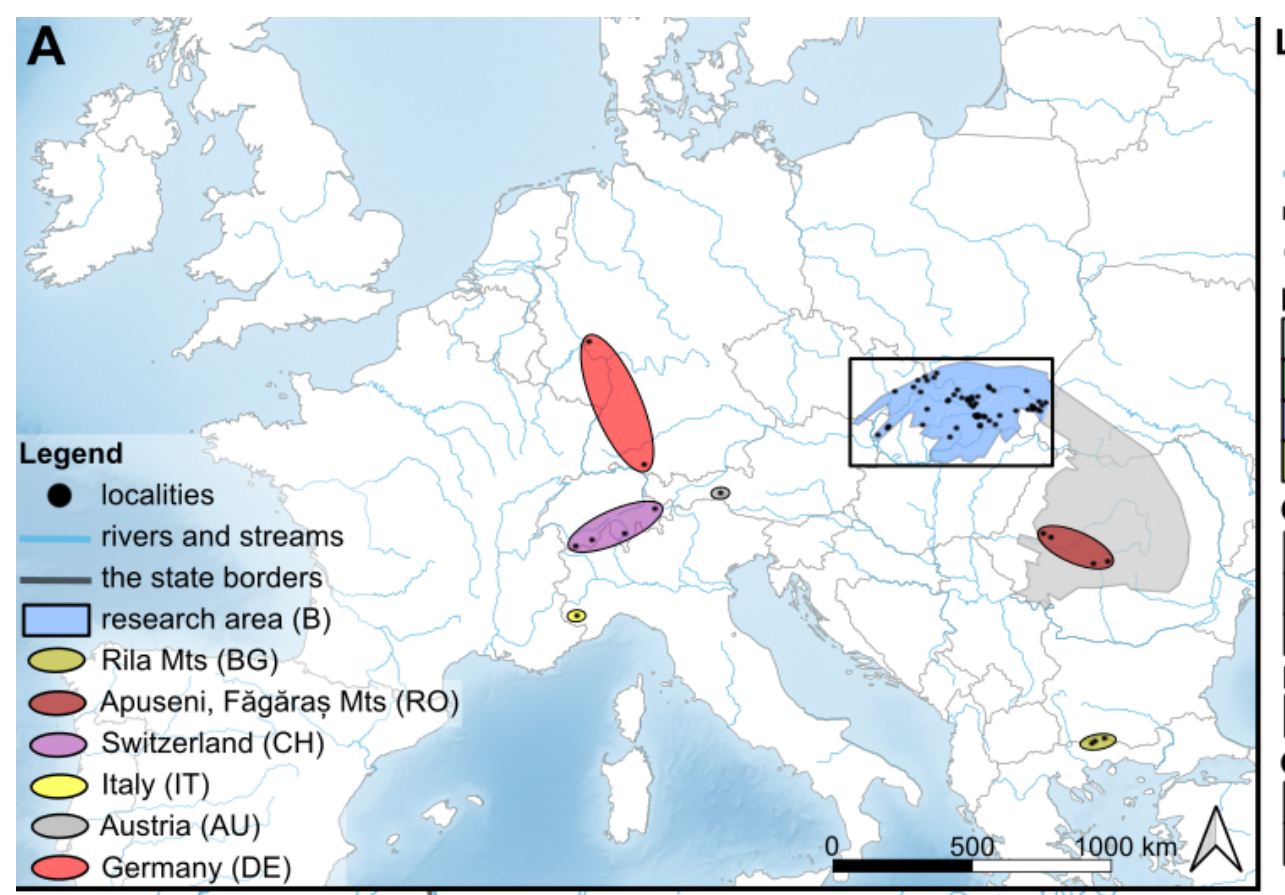

\section{Legend}

- spring localities

stream localities

rivers and streams

Inner/Outer and W/E Carpathians border

the state borders

Inner Western Carpathians

Fatra Tatra Area (FTA)

Slovak Cental Mts (SCM)

Slovak Ore Mts (SOM)

Slanské Hills $(\mathrm{SH})$

Outer Western Carpathians

Western Beskids (WB)

Podhale-Magura Area (PMA)

Slovak-Moravian Carpathians (SMC)

Inner Eastern Carpathians

Vihorlat Mts (VM)

Outer Eastern Carpathians

Poloniny Mts (PM)

Low Beskids (LB)

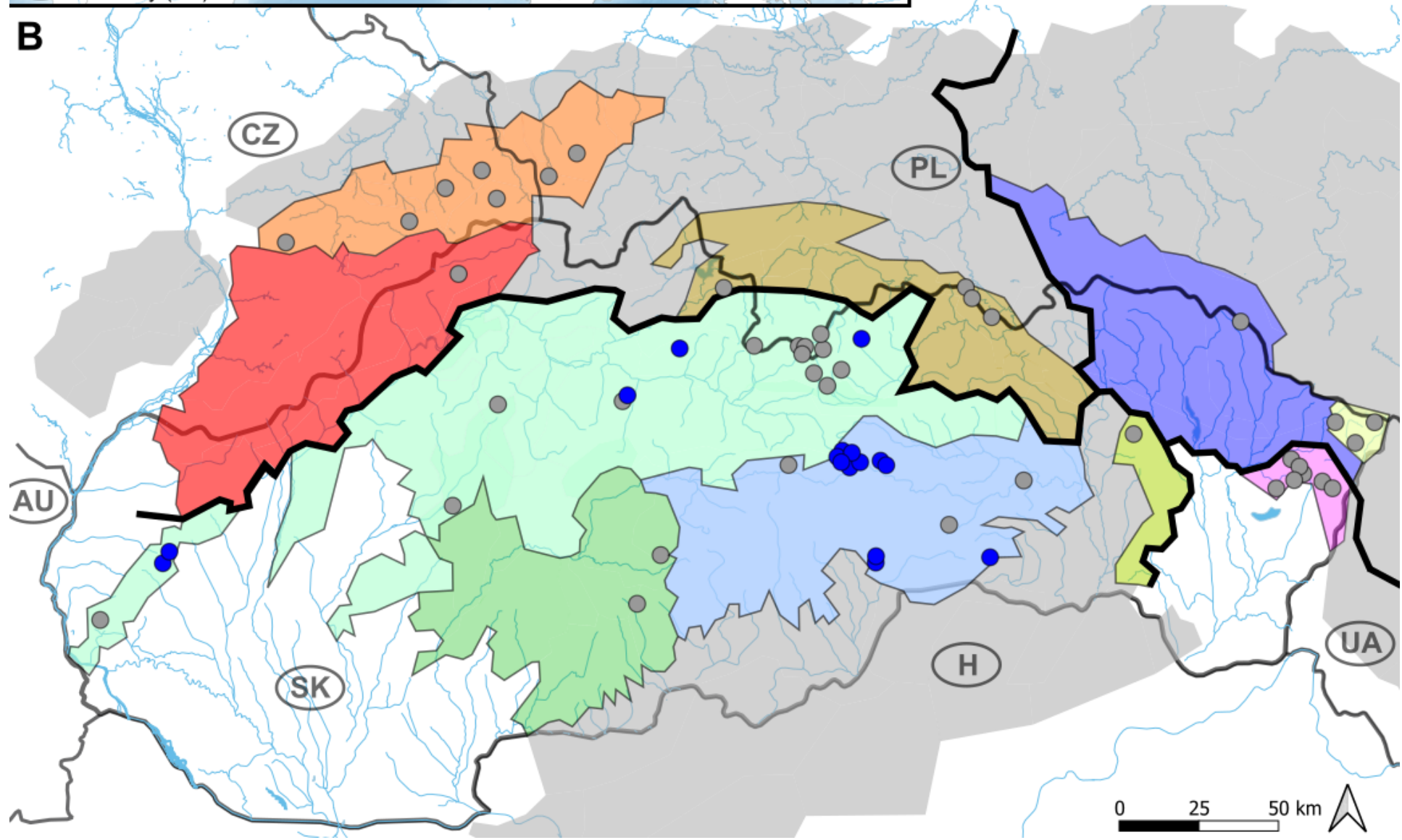

Figure 1

Distribution of the R. tristis sites. (A) Map of the research area (rectangle) within Europe and 15 localities from outside of the research area (ellipses); (B) Research area with 58 sampling sites (15 springs, 43 streams) divided into ten geomorphological units represented by different fill colours (see legend). Abbreviations: Slovakia (SK), Hungary (H), Ukraine (UA), Poland (PL), Czech Republic (CZ), Austria (AU), Bulgaria (BG), Romania (RO). 


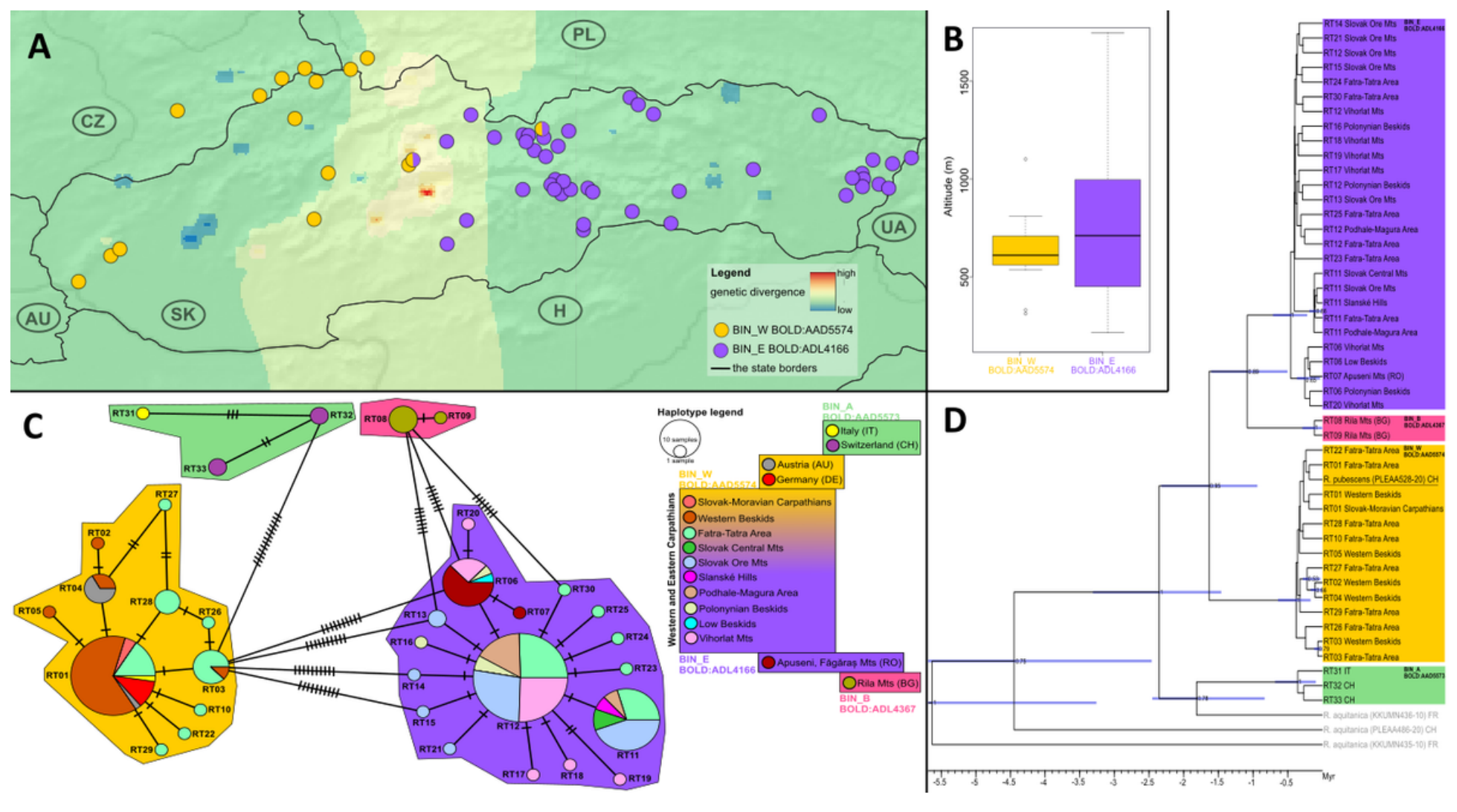

Figure 2

The distribution of the R. tristis BINs and their genetic diversity in the studied area. (A) The genetic landscape map of the studied area based on 5' COI mtDNA haplotypes with the distribution of two different BINs (BIN_W - yellow, BIN_E - violet). Abbreviations: Slovakia (SK), Hungary (H), Ukraine (UA), Poland (PL), Czech Republic (CZ) and Austria (AU); (B) The altitude range of both BINs. The boxplots show the distribution of the altitude above sea level for BIN_W and BIN_E. The boxes represent the interquartile distances (IQD), while the central lines through each box show the medians. The dots indicate outliers and the whiskers extend to the extreme values of the data, calculated as $\pm 1.5 \times$ IQD from the median. Wilcoxon signed-rank test supported the significant differences in altitude range between two BINs ( $p$-value < 0.05); (C) Median-Joining haplotype network (5' COI mtDNA) showing the relationships among haplotypes RT01 - RT33 (including available haplotypes from outside of the studied area). Groups of haplotypes form four BINs (BIN_W, BIN_E, BIN_B, BIN_A), which are colour-coded in the haplotype network according to the legend; (D) Bayesian phylogenetic reconstruction of R. tristis based on $\mathrm{COI} 5^{\prime}$ region. Numbers near nodes are BPP of BEAST v2.6.1. Node bars are $95 \%$ HPD of the divergence time. The outgroup consisted of the European congeneric species R. aquitanica. 


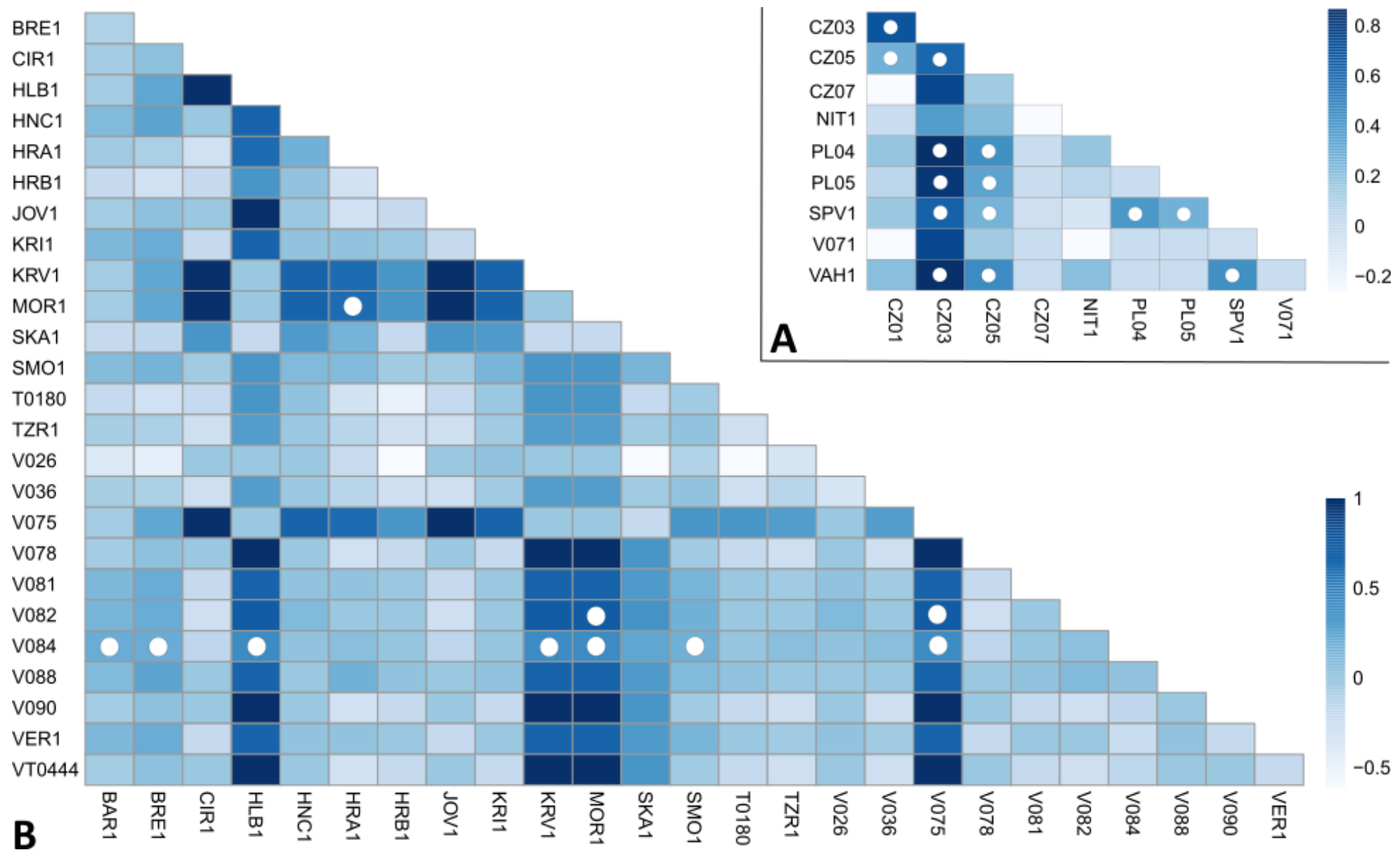

\section{Figure 3}

Heat map of pairwise FST values among studied sites (subpopulations) of R. tristis in the W Carpathians, (A) BIN_W, (B) BIN_E. Darker blue shades indicate higher FST values (as displayed on the bar right of the map). White dots indicate FST $p$-values significantly different from zero) $p$-value $<0.05$ ). 

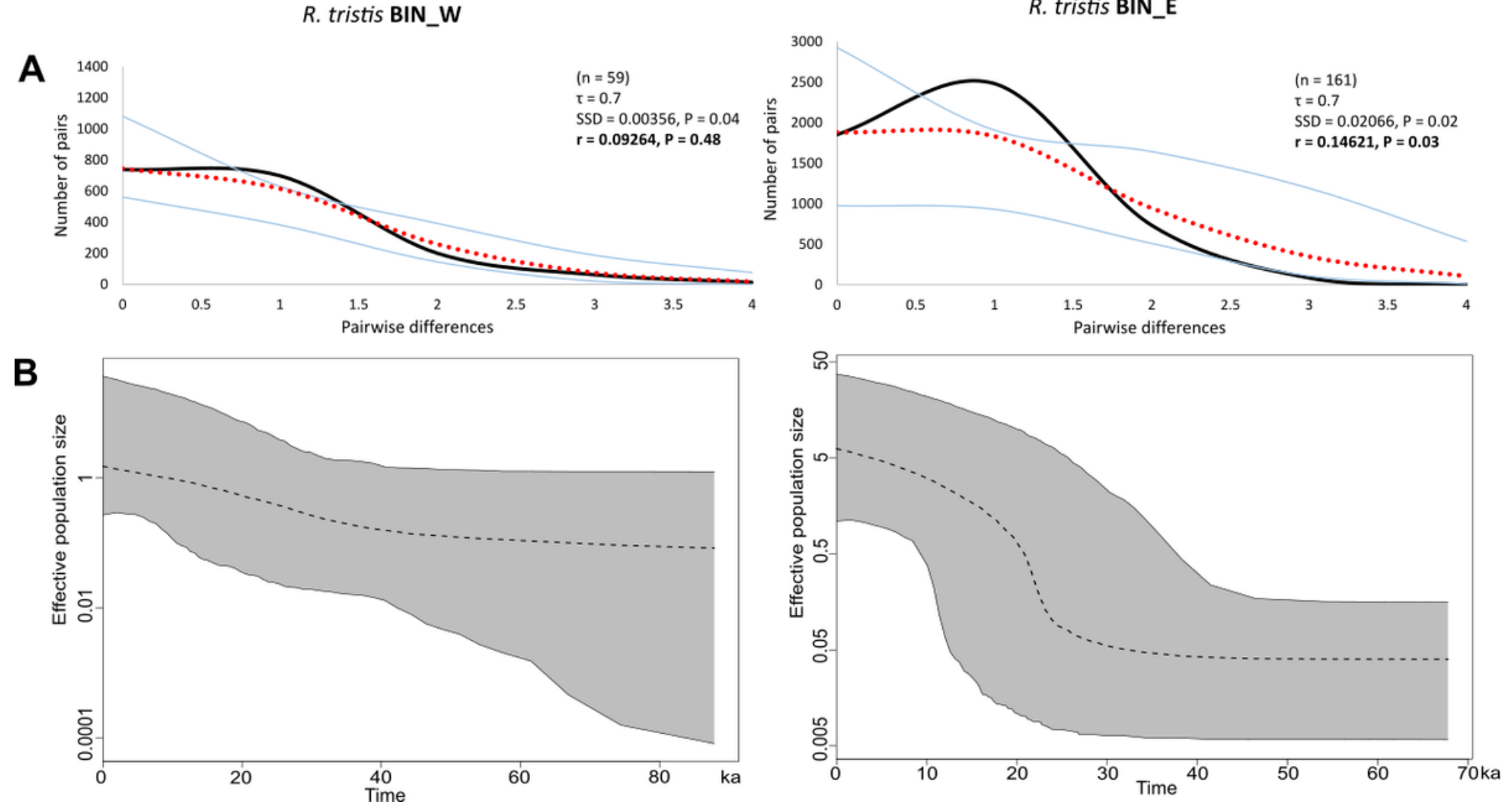

Figure 4

Historical demography of R. tristis populations in the Western Carpathians. (A) Observed unimodal mismatch distribution of BIN_W and BIN_E suggests a recent population expansion. Each plot shows the number ( $\mathrm{Y}$ axis) of pairwise nucleotide site differences ( $\mathrm{X}$ axis) among sequences for each BIN. The fit to the demographic expansion model is evaluated by the SSD and the Harpending's raggedness index ( $r$ ). The solid black line corresponds to the observed frequency of pairwise differences, the dotted red line represents the pattern expected under a model of sudden demographic expansion. The blue lines are the upper and lower boundaries of the $95 \%$ confidence interval; (B) Changes in effective population size through time estimated by Bayesian Skyline plots, reconstructing the population size history using an evolutionary rate 0.0115 substitutions/site/Myr. The $\mathrm{x}$-axis is depicted on a scale of thousands of years $(\mathrm{ka})$, while $\mathrm{Y}$-axis corresponds to the mean effective population size. The dotted line represents the mean, while grey-shaded areas encompass $95 \%$ highest posterior density (HPD). 

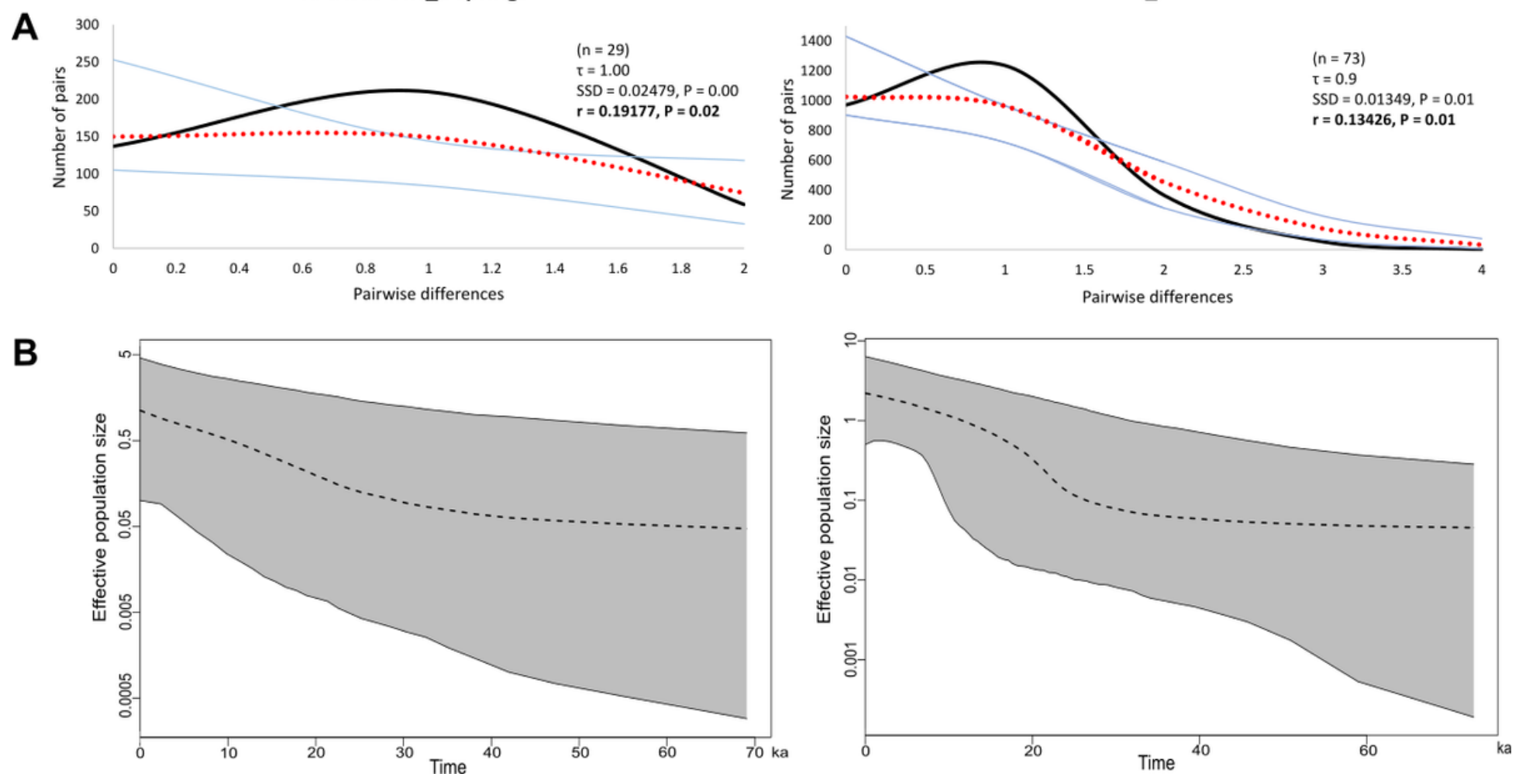

\section{Figure 5}

Comparison of historical demography of R. tristis BIN_E sequences between springs and streams in the W Carpathians. (A) Observed mismatch distributions among springs and streams of BIN_E show the influence of a recent population expansion. Each plot shows the number ( $Y$ axis) of pairwise nucleotide site differences ( $X$ axis) among sequences for each habitat type. The fit to the demographic expansion model is evaluated by the SSD and the Harpending's raggedness index ( $r$ ). The solid black line corresponds to the observed frequency of pairwise differences, the dotted red line represents the pattern expected under a model of sudden demographic expansion. The blue lines are the upper and lower boundaries of the $95 \%$ confidence interval; (B) Changes in effective population size estimated by Bayesian Skyline plots, reconstructing the population size history using an evolutionary rate 0.0115 substitutions/site/Myr. The x-axis is depicted on a scale of thousands of years (ka), while Y-axis corresponds to the mean effective population size. The dotted line represents the mean, while greyshaded areas encompass $95 \%$ highest posterior density (HPD).

\section{Supplementary Files}

This is a list of supplementary files associated with this preprint. Click to download.

- SupplementaryMaterial.docx 